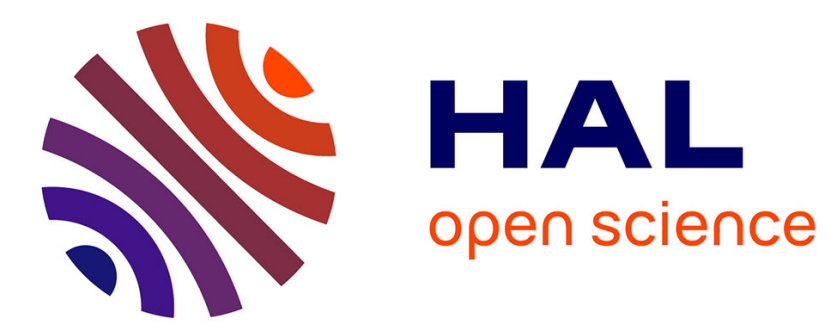

\title{
Production/maintenance cooperative scheduling using multi-agents and fuzzy logic
}

Thierry Coudert, Bernard Grabot, Bernard Archimède

\section{To cite this version:}

Thierry Coudert, Bernard Grabot, Bernard Archimède. Production/maintenance cooperative scheduling using multi-agents and fuzzy logic. International Journal of Production Research, 2002, vol. 40, pp. 4611-4632. 10.1080/00207540210159545 . hal-00767647

\section{HAL Id: hal-00767647 \\ https://hal.science/hal-00767647}

Submitted on 20 Dec 2012

HAL is a multi-disciplinary open access archive for the deposit and dissemination of scientific research documents, whether they are published or not. The documents may come from teaching and research institutions in France or abroad, or from public or private research centers.
L'archive ouverte pluridisciplinaire HAL, est destinée au dépôt et à la diffusion de documents scientifiques de niveau recherche, publiés ou non, émanant des établissements d'enseignement et de recherche français ou étrangers, des laboratoires publics ou privés. 


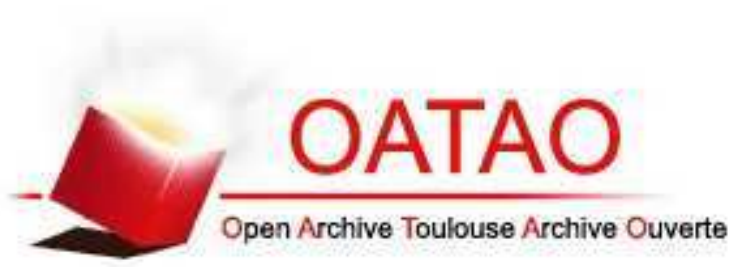

\section{Open Archive Toulouse Archive Ouverte (OATAO)}

OATAO is an open access repository that collects the work of Toulouse researchers and makes it freely available over the web where possible.

This is an author-deposited version published in: http://oatao.univ-toulouse.fr/ Eprints ID: 6881

To link to this article: DOI:10.1080/00207540210159545

http://dx.doi.org/10.1080/00207540210159545

\section{To cite this version:}

Coudert, Thierry and Grabot, Bernard and Archimède, Bernard Production/maintenance cooperative scheduling using multi-agents and fuzzy logic. (2002) International Journal of Production Research, vol. 40 ( $\left.\mathrm{n}^{\circ} 18\right)$. pp. 4611-4632. ISSN 0020-7543

Any correspondence concerning this service should be sent to the repository administrator: staff-oatao@inp-toulouse.fr 


\section{Production/maintenance}

cooperative scheduling using multi-agents and fuzzy logic

T. Coudert, B. Grabot \& B. Archimède

Version of record first published: 14 Nov 2010. 


\title{
Production/maintenance cooperative scheduling using multi-agents and fuzzy logic
}

\author{
T. COUDERT $\dagger$, B. GRABOT ${ }^{\dagger *}$ and B. ARCHIMÈDE $\dagger$
}

Within companies, production is directly concerned with the manufacturing schedule, but other services like sales, maintenance, purchasing or workforce management should also have an influence on this schedule. These services often have together a hierarchical relationship, i.e. the leading function (most of the time sales or production) generates constraints defining the framework within which the other functions have to satisfy their own objectives. We show how the multiagent paradigm, often used in scheduling for its ability to distribute decisionmaking, can also provide a framework for making several functions cooperate in the schedule performance. Production and maintenance have been chosen as an example: having common resources (the machines), their activities are actually often conflicting. We show how to use a fuzzy logic in order to model the temporal degrees of freedom of the two functions, and show that this approach may allow one to obtain a schedule that provides a better compromise between the satisfaction of the respective objectives of the two functions.

\section{Introduction}

Within industrial companies, several functions, often called Departments or Services, have very close links with production at the operational level, even though their respective objectives may be partially conflicting. These functions can be the following.

- Sales: main objective of which is to provide the customer with the right product at the right moment.

- Distribution: objective of which is to respect the product due date and minimize the distribution cost.

- Maintenance: aims to insure the availability of the production resources through curative, and also preventive or cleaning activities.

- Inventory management: has to make raw materials and components available for production.

- Purchasing management: its mission is to buy the resources and services required by the company.

- Human Resource management: one of its operational objectives is to manage the workforce according to technical (skill) and legal (working time) criteria.

These functions are in some cases linked by the product flow, since they may act at different steps of the process elaboration. It is, for example, the case for Purchasing, Inventory Management, Production and Sales, which are sequentially involved. Nevertheless, their respective actors may have different ideas on the optimality of

$\dagger$ LGP/ENIT, 47, Avenue d'Azereix-BP 1629, F-65016 Tarbes Cedex, France.

* To whom correspondence should be addressed. e-mail: bernard@enit.fr 
the flow that they manipulate. The link between functions may also result from the use of common resources, like Production and Human Resource management (which both concern the workers), or Production and Maintenance (acting on the manufacturing machines).

Like in any control problems, the possible conflicts may be avoided by a hierarchical coordination, or solved by negotiation. Nevertheless, note that in practise, the respective degrees of freedom of the functions are determined at the middle term and are seldom set into question at an operational level like the scheduling level. Lot sizes, inventory levels, teams or periods of maintenance are so defined once and belong to the set of hypothesis which constrain the schedule.

In that context, it is paradoxical that even if scheduling has been recognized for a long time as a multi-objective process (Smith 1992), it is seldom admitted that other functions should interfere with production during the schedule elaboration. In practise, the objectives taken into account are usually only related to the customer's satisfaction ('external' or 'exogenous' objectives, related to due date, quality or service) or aim at satisfying internal constraints of the production system ('internal' or 'endogenous' objectives: minimize work in progress, optimize resource use, etc.) (Zülch et al. 1994, Grabot 1998).

Scheduling techniques like constraint-based analysis (Fox 1987) could allow one to model and dynamically take into account objectives coming from other functions while performing the schedule, but the division between functions seems still to be an obstacle at the operational level. Besides, this division is often considered as preventing an improvement of the manufacturing efficiency, and process-oriented reorganization techniques like Business Process Reengineering (Hammer and Champy 1994) are increasingly more widely spread.

Even in standard function-based organizations, we think that important improvements can be obtained by providing the actors with efficient operational tools which could manage the negotiation process, as long as the objectives of each function have been clearly expressed. We have chosen the multi-agent paradigm for the definition of such tool because of its well-known ability to support distributed decision-making. The prototype system that has been developed illustrates the possible cooperation of Maintenance and Production while performing a schedule: these functions are often a source of conflict in companies (e.g. Weinstein and Chung 1999) since, as stated above, they both act on the manufacturing machines. Moreover, reducing the work-in-progress requires that the machines are available when they are required. Tight links should thus be introduced between Production and Maintenance (Swanson 1999).

A panorama on related research is given in Section 2: some relevant studies on multi-agent systems for scheduling are discussed, then some approaches aimed at making Production and Maintenance cooperate are described. Our implementation framework is based on an already existing multi-agent scheduler, the RAMSES system, which is described in Section 3. RAMSES II, the suggested Production/ Maintenance scheduler, is described in Section 4. To allow negotiation, the degrees of freedom of the negotiating partners must be expressed in the same formalism. Section 5 shows that fuzzy sets allow one to define the temporal degrees of freedom of Production and Maintenance as fuzzy temporal windows, allowing one to solve conflicts through a negotiation process which is described. The results of this negotiation-based approach are described in Section 6 . 


\section{Panorama on related research}

\subsection{Multi-agent approaches for scheduling}

The multi-agent paradigm offers a convenient framework for modelling the various entities that constitute the manufacturing system: an agent class can represent a type of entity of the system (machines, orders, workers, etc.) and allows to encapsulate the behaviour of each entity (objectives, reaction to events, etc.). The schedule performance emerges then from the local behaviour of the involved agents, allowing distributed decision-making and reaction capacities. A state-of-the-art of multi-agent approaches for intelligent manufacturing can be found in (Shen and Norrie 1999), which describes more than 30 different studies among which many are oriented on scheduling. Most of these approaches are based on the Contract-Net communication protocol (Smith 1980). Using the Contract-Net, an announcement concerning a task to perform is sent within the multi-agent system. Bids are made by the agents capable of performing the task. The bids are collected and compared, and a contract is made with the agent which has made the best proposal. YAMS (Yet Another Manufacturing System) (Parunak 1987) is certainly the first attempt of using the Contract-Net for task allocation within a manufacturing system. The production system is considered as a hierarchy of manufacturing cells, each node being modelled by an agent. YAMS does not directly allow to generate a schedule, but can repair an existing solution set into question by the occurrence of a disturbance. A multi-agent scheduler, also based on the Contract-Net approach, has been suggested in (Saad et al. 1996). Agents model the articles to manufacture and the machines, the manufacturing objectives being to minimize resource use and cycle time, and to meet the due dates. When an article enters the system, the corresponding agent makes an announce concerning its manufacturing operations. For each operation, bids are made by the capable machine-agents, including a beginning date and the list of operations already planned on the machine. The article-agent chooses the machine which agent suggests the earliest beginning date.

The Contract-Net protocol is again used in Metamorph (Maturana et al. 1999): agents model one more time products and resources, and are coordinated by higher level agents which can keep trace of the machines which bids have not been selected. If a disturbance occurs, a reaction can be defined using these machines.

Other approaches, like Liu and Sycara (1994), suggest a coordination mechanism requiring the definition of the scheduling problem as a constraint satisfaction problem. Resource-agents express the capacity constraints, whereas job-agents express the precedence constraints between tasks, so that the earliest beginning and delivery dates. A similar method is suggested in Miyashita (1998) with the difference that each agent addresses its own constraint satisfaction problem separately from the others. A negotiation process then solves the conflicts.

In other studies, the multi-agent approach is used to repair a schedule (e.g. Tranvouez et al. 1998) or for real-time control of flexible cells (Sohier et al. 1998). In this last case, the interaction between static agents modelling the production environment (robots, machines, etc.) and dynamic agents (modelling parts, tools or Numerical Control programs) allows one to generate a production scenario.

These studies do not all have the same goals, and differences may be noticed between the communication or negotiation protocols which are used. Nevertheless, they have some important common points. 
- In most of these approaches, the agents that model the parts have in charge the respect of the 'external' manufacturing objectives, i.e. those related to the customer's satisfaction, whereas the agents modelling the resources have in charge the respect of the 'internal' objectives, i.e. objectives expressing the need to produce at best cost.

- Approaches using constraint propagation do not usually allow one to add or remove constraints during the solving process, having for consequence the definition of a rather rigid framework. An advantage is that their global approach may lead to an optimal solution.

- Contact-Net offers a rather flexible way to manage the degrees of freedom that may exist in the choice of the resource to perform an operation. A drawback is that the method is myopic, i.e. the choice of a resource is usually based on a local performance evaluation.

- In all the cases, the negotiation process must be carefully controlled: many resource conflicts are usually to be solved during the schedule elaboration, and solving these conflicts through negotiation can be time consuming. Therefore, it is important to only introduce negotiation where it brings an added value.

\subsection{Cooperation between production and maintenance}

Several types of Maintenance are to be distinguished since they lead to different kinds of interactions with Production (e.g. Paz and Leigh 1994). Their classification may vary according to the chosen criterion, but the following categories are usually defined.

- Curative maintenance occurs after a machine breakdown. In that case, there is no conflict between Production and Maintenance since the concerned machine is not anymore available for Production. Nevertheless, conflicts may arise between several manufacturing resources when they require at the same time a curative maintenance activity. This type of conflict may be considered as internal to the Production function and is usually solved thanks to an implicit of explicit level of priority between machines requiring a curative maintenance activity.

- In preventive maintenance, the decision to perform an activity precedes the occurrence of disturbance. Three main preventive maintenance categories may be distinguished:

- Systematic preventive maintenance, characterized by a periodicity between maintenance activities, determined arbitrarily or according to the resource law of behaviour.

- Condition-based maintenance, triggered by the occurrence of symptoms or by reaching a given level of damage.

- Predictive maintenance, based on the occurrence of events that may lead to disturbances.

Scheduling maintenance activities is a problem on its own: the duration of the maintenance activities may be imprecisely known (Paz and Leigh 1994) and the beginning date of a preventive maintenance activity may be brought forward or postponed in comparison with the optimal date (Gits 1994). Many studies have been done on the composition of maintenance teams, like Basker and Husband (1982) and Barnett and Blundell (1981). The efficiency and cost of maintenance 
policies are also often compared (Paté-Cornell et al. 1987, De Carvalho and Noyes 1996, Kelly et al. 1997).

Three possible hierarchical positions of Production and Maintenance can be used in industrial systems.

- Production can have a higher position, with the result that preventive maintenance is only performed during under-loaded periods. This can be the case when a high utilization ratio of the machines is required, especially when the machines are easy to maintain.

- Maintenance can have the higher position: preventive maintenance activities are positioned first and define closed periods in the machine calendars. The manufacturing operations are scheduled as a second step.

- Production and Maintenance can be considered as having the same hierarchical level, requiring either coordination or cooperation. This cooperation or coordination is particularly needed when the periods of preventive maintenance are short regarding the manufacturing processing times, and/or when the maintenance activities are long in comparison with the manufacturing operations.

In that context, the coordination or cooperation between Maintenance and Production has several objectives.

- Be sure that enough time is left by Production to Maintenance activities.

- Be sure that Maintenance performs the preventive maintenance activities at the right moment.

- Be sure that Maintenance reacts rapidly to machine breakdowns (Gits 1994, Rishel and Christy 1996, Swanson 1999).

Rishel and Christy (1996) described an approach where Maintenance is considered together with Production in an MRP system: a Material Requirement Planning is performed for the two types of activities and various maintenance policies are tested and assessed. Weinstein and Chung (1999) showed a multi-objective linear programming method that performs a hierarchical planning for production and maintenance activities. In Brandolese et al. (1996), a flow-shop system is scheduled, and a probabilistic model taking into account cost and reliability calculates the temporal windows where the maintenance operations should be located. The use of probabilistic approaches has also been considered in order to plan maintenance activities on production lines in Sanmarti et al. (1997) where, in each temporal interval of the simulation horizon, a model allows one to choose between producing and having the risk to see a failure occur and performing a maintenance activity and making the cost increase. A similar approach is used in Ashayeri et al. (1996): the maintenance activities are modelled as fictive manufacturing orders (MOs) and scheduled together with manufacturing operations. In Deniaud et al. (1999), an industrial case is considered in which simulation is used for balancing maintenance and manufacturing activities.

In all these works, it can be seen that a higher level coordination is defined, which may prevent a good reactivity of the system. On the other hand, this coordination is less time consuming than cooperation when a schedule is performed.

More generally, in Ayel (1994) can be found the description of a multi-agent system where each agent represents a decision centre of the production system. The various agents may then represent different functions and the possible conflicts 
between functions are solved by negotiation. Nevertheless, the purpose of this multiagent system is mainly to synchronize different activities and not to coordinate different functions acting on the same activity. Consequently, the results can hardly be adapted to the problem of cooperative scheduling.

As stated above, multi-agent systems may provide an efficient paradigm for modelling negotiation activities between different entities and have efficiently been used in scheduling. We describe below how an existing multi-agent scheduling system has been modified to provide a cooperation medium for scheduling maintenance and production activities.

\section{RAMSES multi-agent scheduler}

The RAMSES (ReActive Multi-agent SystEm for Scheduling) environment has been developed to test various scheduling strategies in a multi-agent context (Archimède and Coudert 2001). It is quite open and flexible and allows one, for instance, to deal with flexible routings (an operation can be performed by several machines under different conditions, operations can be defined as permutable, etc.) and to associate different objectives to the agents. The RAMSES multi-agent model is based on the architecture described in figure 1.

This communication protocol between agents used in RAMSES is close to the Contract Net protocol. At the beginning of the scheduling process, the supervisor agent creates and initializes the MO and machine agents. The possible objectives of a MO agent are to minimize its cycle time, to respect a due date or to minimize a manufacturing cost (the machines can have different hourly rates). The supervisor is in charge of controlling the execution of the negotiation cycles between agents and the access to the blackboard describing the current solution and the bids under negotiation.

A MO agent has to plan manufacturing operations defined in its associated routing. It expresses its requirements by an announce containing the operations, their characteristics (activity, minimum processing times) and an earliest infinite capacity planning which is communicated to the blackboard. This blackboard can be seen as a virtual Gantt chart representing the current state of the negotiation process at different conceptual levels: operations are first associated to activities

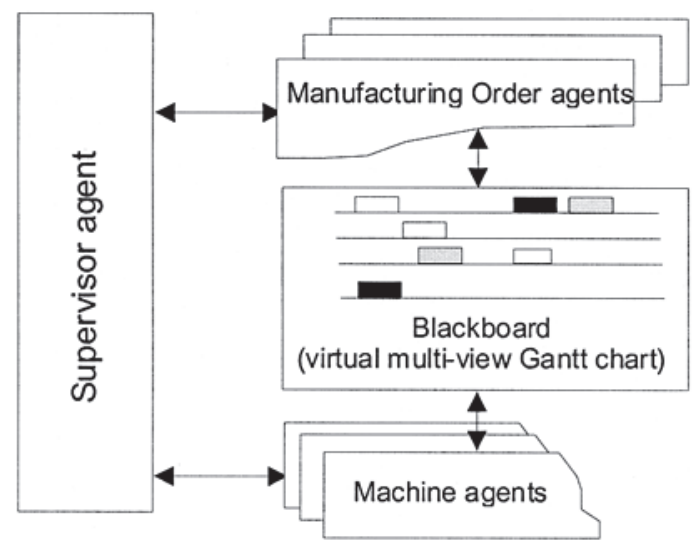

Figure 1. RAMSES architecture. 
(drilling, lathe, etc.), then to machines while some operations are definitively located whereas others can still be moved.

Like in the Contract-Net, the Machine Agents make bids on the operations offered by the MO agents. Each bid includes a correction of the processing time according to the capacity of the machine to perform the activity, and suggests a location of the operation. The particularity of the RAMSES approach compared with the classical Contract Net protocol concerns the nature of the bids. In RAMSES, two different bids are made by each Machine agent, corresponding to an effective and potential position.

- Effective position: takes into account all the other operations that have already been placed on the machine, and also all the operations that the machine has offered to process and which have not yet been accepted by the MO agents. It refers so to a situation in which all the bids in progress would be accepted.

- Potential position: obtained by only taking into account the considered operation. All the other bids in progress are considered as being rejected. Consequently, the effective position gives the worst proposition of a Machine Agent, while the potential position gives its best possible proposition, subject to deterioration through time if bids in progress are accepted.

Each MO agent compares the different bids received from the machine agents to its objectives. The MO agents can accept the effective positions suggested by the machine agents: in that case, the negotiation process ends. If the effective position does not give satisfaction, and if the potential position is much better, the MO agent can take the risk to wait, expecting that this potential position will become effective. In that purpose, the MO agent makes a new bid on the base of the potential position.

Another major difference between RAMSES and the Contract-Net is that in the latter, an order is usually planned operation by operation without any competition with the other orders: the processing sequence of the MOs has so a great influence on the result. In RAMSES, MOs are competing with each other for each operation. Other originalities of RAMSES concern the definition of the routings, e.g. with the concept of activity which provides more degrees of freedom for negotiation. Finally, a global validation process can be used in order to validate at the same time all the operations of a MO, on the base of the effective positions suggested by the machines. A graph is built containing the possible choices for performing the operations, and the most favourable path according to the current objectives (mainly duration and cost) can be adopted in a single step. This possibility permits a more global view on the manufacturing process, which corrects the myopia which is often inherent to the Contract-Net approach.

RAMSES has been implemented in $\mathrm{C}^{++}$on a $\mathrm{PC}$ and uses a standard Windows Interface. It is compliant with the CORBA communication standard and can therefore be remotely used through a network.

The modifications of RAMSES allowing a Production and Maintenance cooperation are detailed below.

\section{RAMSES II: a production/maintenance multi-agent scheduler}

\subsection{RAMSES_Maintenance multi-agent system}

As stated above, scheduling maintenance activities cannot be directly assimilated to scheduling manufacturing activities. As a first step, a RAMSES_Maintenance 
multi-agent system has so been derived from the RAMSES system. This multi-agent system is dedicated to the schedule of maintenance operations and has required the following changes:

Several types of 'maintenance orders' can be planned.

- A preventive maintenance order is characterized by a preferred temporal window, whose definition is discussed in Section 5.2. It is associated to one or several maintenance activities defining a maintenance routing. Each maintenance activity requires maintenance resources (operators and/or tools).

- A condition-based maintenance order is created according to a condition related to the schedule of manufacturing operations (schedule of a soiling activity, schedule of a given sequence of manufacturing activities, etc.). This type of order is not associated to a temporal window and requires the RAMSES_Maintenance system to be connected to a RAMSES_Production system.

- A corrective maintenance order is associated with a machine. It is characterized by an earliest beginning date and composed of one or several maintenance activities. A priority level can also be considered if available. A corrective maintenance order is only planned in the case of reactive scheduling when a machine breakdown occurrence has been reported. It will only be used if the RAMSES_Maintenance is connected with a RAMSES_Production, which is also used for executing the schedule.

In an autonomous mode, the maintenance supervisor agent activates the maintenance order agents, which write on the blackboard the announcement concerning the operations they need to perform. The possible maintenance resource agents make bids on these announces with a process similar to the RAMSES_Production one. The main difference is that the maintenance order agents consider the respect of the temporal windows as performance criteria for assessing the bids and not the due dates like in RAMSES.

\subsection{RAMSES II: connection of RAMSES_Production and RAMSES_Maintenance systems}

A RAMSES II scheduler is composed of several RAMSES_Production and RAMSES_Maintenance multi-agent systems, which blackboards are actualized during the negotiation process. Each RAMSES_Production system represents a workshop, and each RAMSES_Maintenance system represents a set of maintenance resources (for instance, a team). Within the Maintenance multi-agent system, a maintenance agent is created for each manufacturing machine. For simplification, we shall illustrate the system behaviour on the case of one production and one maintenance system. Two new types of agents have been added to the system.

- 'User agent', in order to allow a direct action of the human user on the scheduling process. The possible actions that can be performed through the user agent concern changes in the rules that define the behaviour of the Machine agents, changes in the objectives of the involved agents or relaxation of different types of constraints. The user agent also allows one to describe a disturbance occurring while the schedule is executed.

- 'Negotiator agent', in charge of the negotiation process. This agent does not have a higher hierarchical position than the maintenance and production 
agents but aims at accelerating negotiation by gathering the data coming from the two systems. The definition of the compromise between the requirements expressed by the maintenance and production agents, contained in the Negotiator, can be modified by the User agent.

When only systematic preventive maintenance activities and manufacturing operations are considered, the negotiation process is performed as summarized in figure 2 .

- When a manufacturing operation is to be scheduled by a machine agent, a temporal window showing the earliest and latest possible beginning date for the operation is calculated. Details on these temporal windows are given in Section 5. The information is sent to the Negotiator (message (1)).

- Negotiator asks the Maintenance agent of the considered machine whether maintenance activities could interfere with the temporal window of the manufacturing operation (message (2)).

- Maintenance agent sends the list of the concerned Maintenance activities with their temporal windows (message (3)).

- Negotiator checks whether there is a real conflict between the manufacturing and maintenance operations. If a position of the operations allows to satisfy the constraints expressed by the temporal windows, there is no conflict, and the Negotiator asks the Machine agent to plan the operation according to this position (message (4)).

- If there is a real conflict, i.e. the positions cannot give full satisfaction, the Negotiator suggests a compromise and asks the Machine agent to schedule the manufacturing operation at the suggested beginning date (message (4)) and asks the Maintenance agent to reschedule the Maintenance activities at the suggested beginning date (message (5)).

When condition-based maintenance activities are considered, the role of the Negotiator is slightly modified: if a manufacturing operation has to be scheduled, the Negotiator checks whether the condition for creating a condition-based maintenance activity is verified. If yes, the Negotiator asks the Maintenance agent to

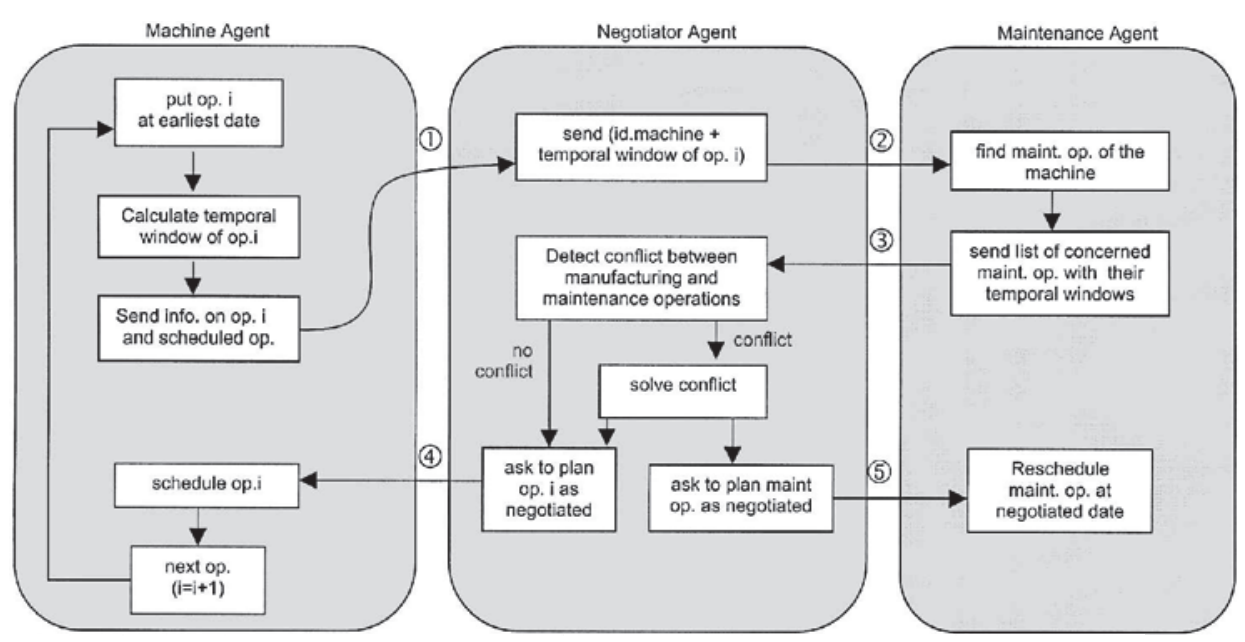

Figure 2. Cooperation/negotiation between maintenance and machine agents. 
create the Maintenance order, then to calculate its temporal window. Conditionbased maintenance orders can be strict, i.e. no manufacturing operation can be scheduled on the machine before the maintenance operation, or may accept a given tolerance on their positioning. This tolerance is described in the temporal window attached to the condition-based maintenance order. If the Maintenance order is mandatory, there is no conflict since it is planned first. Otherwise, the global negotiation process is performed as explained above.

In the first version of RAMSES II, the temporal windows of the operation s have been defined as crisp intervals (Coudert et al. 1999). The satisfaction provided by the position of a maintenance activity was as a consequence binary: full satisfaction if the operation was beginning within the window, null satisfaction if not. As discussed above, this is not very consistent with the location of preventive maintenance activities, which is subject to preferences, but is seldom mandatory. For instance, it is clear that being early or late by a few hours is not very important for a monthly maintenance operation. A well-known interest of fuzzy logic is to cope with the problem of arbitrary thresholds: we explain below how the system behaviour has been made more realistic by modelling the temporal degrees of freedom of the operations by fuzzy windows.

\section{Modelling degrees of freedom and conflict solving in RAMSES II}

The idea of defining relaxable temporal constraints by fuzzy sets has often been used in scheduling, from Kerr and Walker (1989) to Ishii (2000). We shall see below how fuzzy temporal windows can be defined for due dates, manufacturing operations and maintenance operations. The degree of satisfaction attached to the location of an operation (maintenance or manufacturing) will provide a way to assess a compromise, which is a mandatory condition for an efficient management of the negotiation process.

\subsection{Fuzzy modelling of a due date}

The modelling of the preference associated to the respect of a due date ' $a$ ' by a fuzzy set was first introduced by Kerr and Walker (1989) and it is now a classic in fuzzy scheduling. We have chosen to represent a fuzzy due date satisfaction as described in figure 3, expressing that the order may be early (full satisfaction, i.e. $\left.\mu(t)=1 \forall_{t}<a\right)$ and that a delay becomes progressively unacceptable (satisfaction from $\mu(t)=1$ to $\mu(t)=0$ for $a<t<b)$. In figure 3 , the last manufacturing operation is represented by a hatched rectangle: in that example, the satisfaction of the due date constraint is $\mu(t)=0.3$.

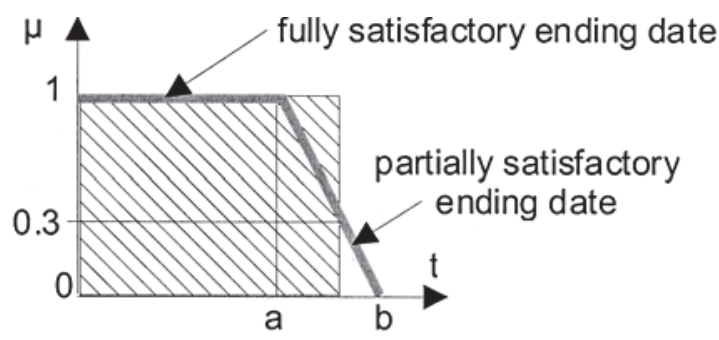

Figure 3. Fuzzy modelling of the preference attached to the due date respect. 
This type of model is more consistent with the industrial reality than a binary evaluation, but in some exceptional cases the due date can of course remain a crisp constraint (departure of a truck or ship, real just-in-time with the customer, etc.).

\subsection{Fuzzy modelling of a maintenance operation}

According to what has been stated in Section 2.2, the preference attached to the beginning date of a maintenance activity can be modelled by the fuzzy set of figure 4 . Starting from the centre, the left slope can be interpreted as a decreasing satisfaction due to an early maintenance that increases the maintenance cost. The right slope can be interpreted as a decreasing satisfaction due to a possible loose of reliability. This fuzzy set allows one to assess the degree of satisfaction associated to the position of a maintenance operation: it is, for example, 0.8 for the activity represented by a grey rectangle in figure 4.

\subsection{Fuzzy modelling of a manufacturing operation}

In our opinion, the fuzzy maintenance temporal windows as described above are a quite realistic extension of the classical maintenance windows. Performing a negotiation process requires one to model the degrees of freedom available for each partner: describing the preference attached to the possible location of a manufacturing operation by a fuzzy set is so necessary. The difficulty is that RAMSES_Production performs a schedule in a way close to list scheduling (when a machine can perform several operations, the machine agent chooses their sequence according to a dispatching rule). Therefore, the system does not define temporal windows for manufacturing operations. Nevertheless, other techniques like analysis under constraints (Erschler et al. 1976) perform a schedule by defining temporal windows for manufacturing operations, then by propagating these constraints in order to find a feasible schedule. Even if this method is not implemented in RAMSES, we have used the same approach in order to define the temporal windows of the manufacturing operations. The window of the $n$th manufacturing operation is defined by placing the previous operations of the routing as early as possible, and the following ones as late as possible. The time between the end of operation $(n-1)$ and the beginning of operation $(n+1)$ defines the temporal window of operation $n$. We could have used these crisp temporal windows in RAMSES II, but the consequence would be an imbalance between the maintenance operations, which location could provide a partial satisfaction, and the manufacturing operations which location would only

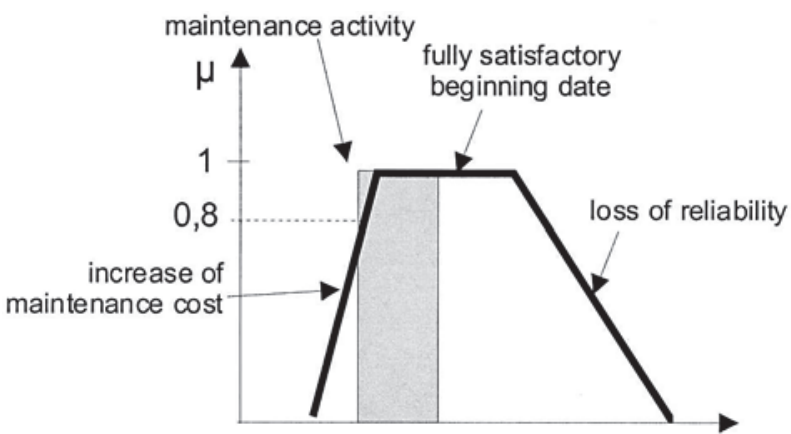

Figure 4. Preferred beginning dates for a maintenance activity. 


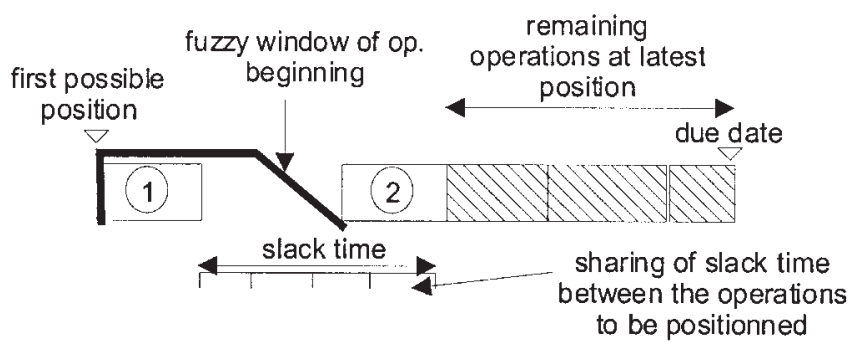

Figure 5. Fuzzy temporal window for the beginning of a manufacturing operation.

be 0 or 1 . What we really need is a way to model to what extent the constraint expressed by the window can be relaxed. Figure 5 shows a way to define this possible relaxation. The hull of the fuzzy set describing the preferred position (i.e. the points for which $\mu(t) \neq 0)$ is the period between the first possible beginning of the operation (place 1 on figure 5) and the last possible beginning, i.e. when the operation is set at the latest possible position, denoted (2)).

Consider the slack time of the operation to be planned, defined as the time left until its due date minus the sum of processing times of the remaining operations of the MO. To determine what positions give complete satisfaction (i.e. the kernel of the fuzzy set, which is the set of points for which $\mu(t)=1)$, let us share the slack time between the operations to be planned (considered operation + remaining ones). In figure 5, three operations plus the considered one remain to be planned. The slack time is divided by 4 , and we consider that the beginning of the manufacturing operation to be planned gives full satisfaction if the operation only consumes its portion of slack time. This slack time is equivalent to the free slack time of the PERT method. If the beginning of the operation is outside the kernel of the fuzzy set, the slack time of the following operations is progressively consumed, even if the due date can still be met. This is the equivalent of the total slack in PERT: the satisfaction provided by this location decreases progressively as far as the total slack is consumed. After position (2) of figure 5, it is not possible anymore to meet the due date: this position is considered as providing a null satisfaction.

If the due date is itself fuzzy, the crisp due date of figure 5 is replaced by the last point of the fuzzy due date (point for which $\mu(t) \neq 0$ ). If it is not possible to meet the due date (end of last operation $>$ due date), we consider that only an immediate beginning of the operation provides satisfaction: the fuzzy temporal window is then reduced to a point. It can be noticed that the upper boundary of the fuzzy window is crisp since it is given by the first possible location of the operation.

Let us now explain more precisely the negotiation process described in figure 2 using these fuzzy temporal windows.

5.4. Conflict solving through negotiation in the presence of fuzzy temporal windows

First, the maintenance agents must be provided with a method to allow them to detect conflicts with the manufacturing operation which has to be planned. With the notation shown in figure 6 , the condition of conflict is that $(\mathrm{b} 1+\mathrm{L} 1>\mathrm{c} 2)$ AND $(\mathrm{b} 2+\mathrm{L} 2>\mathrm{c} 1)$.

The comparison of the possible location of a maintenance activity or manufacturing operation with its temporal window allows us to assess the satisfaction provided by each position. It is then necessary to define an operator allowing one to 


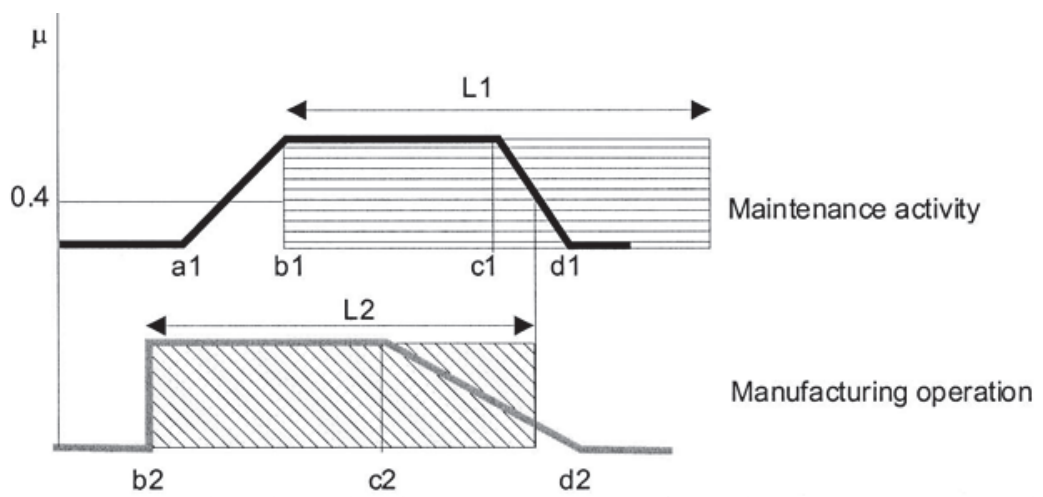

Figure 6. Example of conflict between a maintenance activity and a manufacturing operation.

aggregate these two degrees of satisfaction in order to assess the quality of the compromise defined by these two positions. If we consider as a first step that maintenance and production have the same priority, the choice of an aggregation operator is firstly constrained by conditions on limits:

- if the two operations have a fully satisfactory position, the aggregated result should be 1;

- if one of the operations has a null satisfaction, the result should be 0 .

Taking the average degree of satisfaction does not fit these requirements, or the maximum operator. The minimum or product operator can be used. The first one has been used here, but other criteria may be defined in order to express more complex relationships between maintenance and production (weighted average value, etc.). Multicriteria optimization can also be of interest in order to address this aggregation problem.

Because of this choice, our goal while trying to find an acceptable position for the maintenance and manufacturing activities is to maximize the minimum degree of satisfaction provided by the two positions.

From figure 6, we can see the following.

- The manufacturing operation can be performed first without having a null satisfaction of the maintenance activity position if $(\mathrm{b} 2+\mathrm{L} 2<\mathrm{d} 1)$. In that case, the optimal position is defined by a manufacturing operation performed as early as possible (beginning at b2) followed by the maintenance activity (beginning at $(\mathrm{b} 2+\mathrm{L} 2)$ ). In figure 6 , the position of the manufacturing operation gives satisfaction with a degree 1 , whereas the position of the maintenance activity gives satisfaction with a degree 0.4 .

- The maintenance activity (denoted MaintAct) can be performed first without having a null satisfaction of the manufacturing operation (ManOp) position if $(\mathrm{a} 1+\mathrm{L} 1<\mathrm{d} 2)$. Since we want to maximize: $M=\min (\mu$ (begin ManOp), $\mu($ begin MaintAct $))$, the best position is obtained when $\mu$ (begin ManOp $)=$ $\mu$ (begin MaintAct). The maintenance activity begins in the ascending slope of the fuzzy set of the top of figure 6 or in its kernel, whereas the manufacturing operation begins in the kernel of the fuzzy set of the bottom of figure 6 , or in its descending slope. 
If the two conditions $((\mathrm{b} 2+\mathrm{L} 2<\mathrm{d} 1)$ and $(\mathrm{a} 1+\mathrm{L} 1<\mathrm{d} 2))$ are satisfied, the two previous solutions must be tested (maintenance first and production first) in order to check which positions provide the best aggregated satisfaction degrees. If none of the two conditions is satisfied, one of the positions will be satisfied with a degree 1, the other with a degree 0 . In that case, it is necessary to ask the user whether the maintenance or the production is to be privileged.

\subsection{Taking into account the capacity of the maintenance resources}

Until now, we have implicitly considered that the resources required to perform the maintenance activities were always available, i.e. we have performed a schedule with finite capacity on the manufacturing resources, but infinite capacity on the maintenance resources.

As explained above, several different maintenance resources can be available in order to perform a required maintenance activity. In that case, the negotiation protocol shown in figure 2 is modified as follows.

- When the maintenance agent identifies that a maintenance activity is required on a machine, it reacts like a MO agent, i.e. it sends an announcement to the maintenance resources describing the type of maintenance activity and the required temporal window.

- Each maintenance resource has its own blackboard, i.e. a virtual Gantt chart showing its periods of occupancy. It can so generate a calendar showing the periods when it can perform the suggested activity. A bid including the calendar of each possible resource is then sent to the Maintenance agent by the Resource agents, which can perform the maintenance activity.

- The bid that best fits the Maintenance objectives is selected. In this first version, the assessment only concerns the respect of the location of the maintenance activity, then the cost (depending of the resource hourly rate). The resource that has the calendar with the largest intersection with the maintenance window is selected. If several resources have a full intersection, the resource with the lower cost is selected. The test of more complex strategies, e.g. based on multicriteria optimization, is in progress.

The temporal window of the selected resource is sent to the Supervisor, which tries to find the best position for the maintenance and manufacturing operations, according to the earlier detailed protocol.

\section{Examples and results}

\subsection{Examples of schedules}

A first example of the result is shown in figure 7 , which was obtained with 25 MOs using two machines on which nine preventive maintenance activities were performed. The upper Gantt chart shows the planning of the Maintenance team (only one team is considered here), while the bottom Gantt chart shows the schedule of the operations on the manufacturing resources. On this chart, manufacturing operations are represented by coloured rectangles and maintenance operations by hatched ones. The two Gantt charts can be visualized on different computers since the negotiation process can be performed through the Net using the CORBA protocol.

The fuzzy sets representing the preference given to the beginning dates of the maintenance operations have been drawn on the upper Gantt chart for clarity, and 


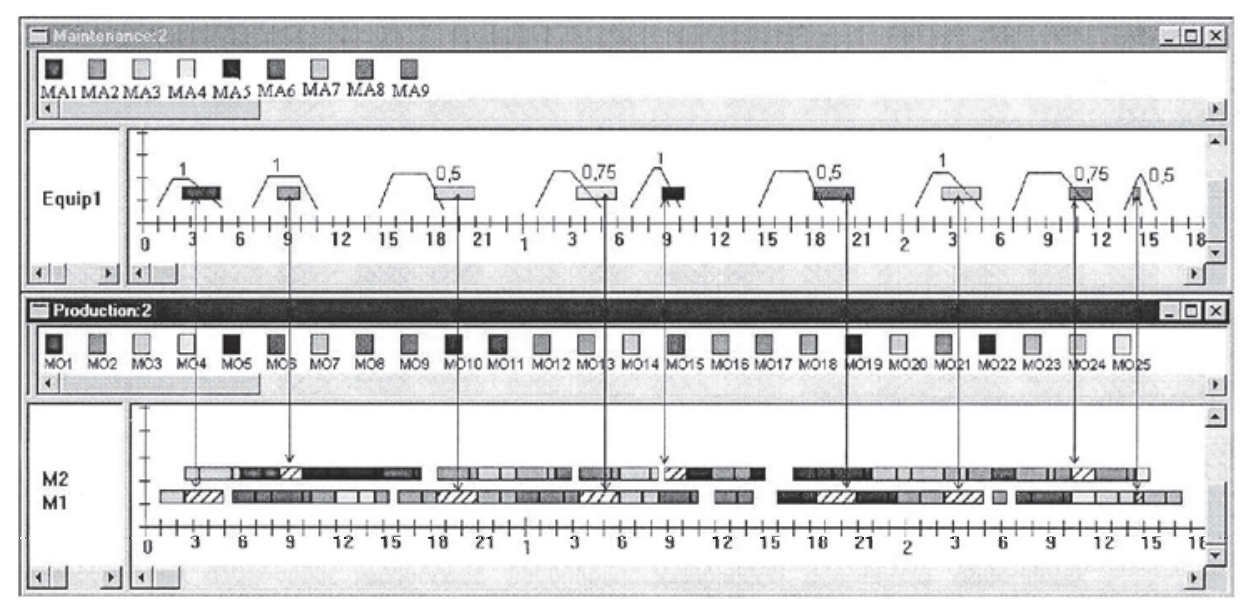

Figure 7. Negotiated schedule of preventive maintenance operations.

the degrees of satisfaction provided by the position of each operation are given. It can be seen in figure 7 that after the negotiation process, the maintenance activities 3 , 4, 6 and 8 have been delayed, while activity 9 is performed earlier than expected. For maintenance activities 1, 2, 5 and 7 , it has been possible to find a location allowing one to keep a satisfaction degree equal to 1 .

The use of condition-based maintenance operations is shown in figure 8. Two operations of 60 minutes and three of 30 minutes have been positioned on the maintenance and manufacturing planning: these operations are conditioned by their following manufacturing activity. Therefore, they stand here for set-up operations. If they are conditioned by their previous operations, they can also model cleaning operations.

A drawback of the method is that the bid and negotiation processes are time consuming. Consequently, the scheduling process is much slower than a classical

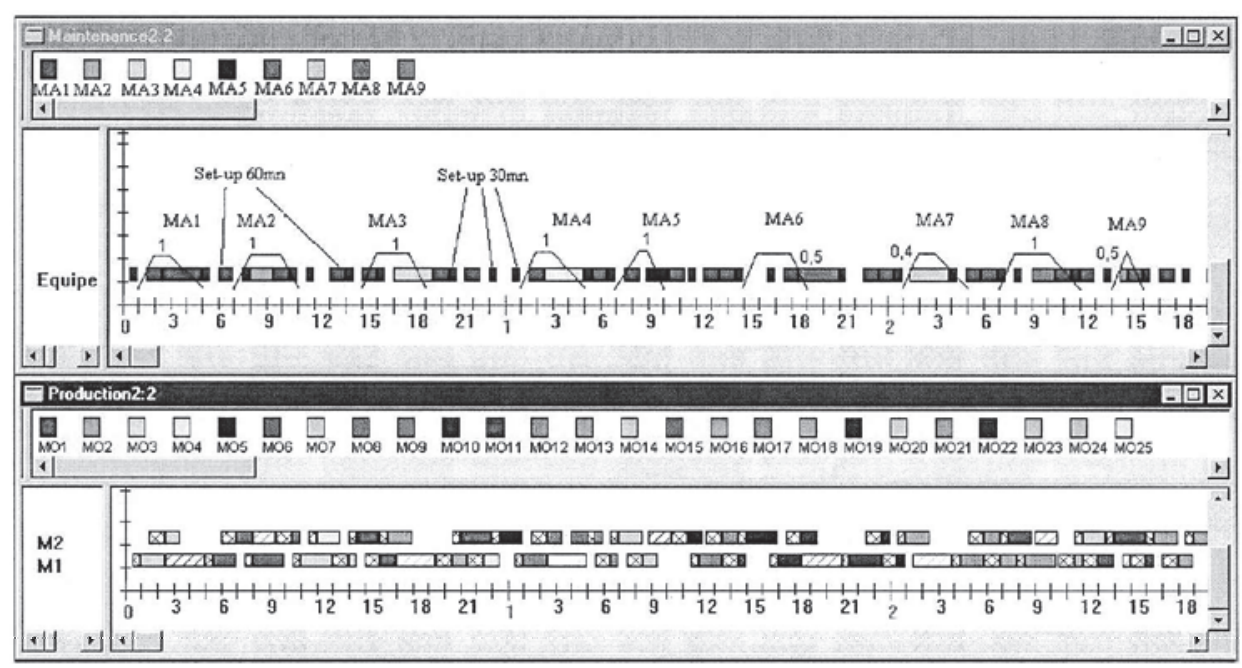

Figure 8. Gantt chart with preventive and condition-based maintenance activities. 


\begin{tabular}{ccc}
\hline Number of activities per machine & Manufacturing & Manufacturing + maintenance \\
\hline 1 & $0: 00: 06$ & $0: 01: 41$ \\
2 & $0: 00: 27$ & $0: 08: 50$ \\
3 & $0: 00: 30$ & $0: 19: 27$ \\
4 & $0: 00: 45$ & $0: 30: 15$ \\
6 & $0: 01: 30$ & $1: 26: 40$ \\
\hline
\end{tabular}

Table 1. Time needed to perform a schedule.

one. The time needed not only depends on the negotiation, which is speeded up by the presence of the negotiator, but also mainly on the number of resources that can perform an activity (manufacturing or maintenance activity), which all send bids in response to an announcement. This will be shown with the following schedule.

- Manufacturing: $50 \mathrm{MOs,} \mathrm{six} \mathrm{machines} \mathrm{in} \mathrm{the} \mathrm{workshop,} \mathrm{six} \mathrm{operations} \mathrm{per}$ routing.

- Maintenance: 24 maintenance activities, one maintenance activity per day for each machine, one maintenance team.

The time needed to perform a schedule in this example according to the number of activities that can be performed by each machine is shown in table 1 . The processor used here is very slow (Pentium $133 \mathrm{MHz}$ ), but the increasing power of the processors makes the comparison between the different processing times more relevant than their absolute values.

Table 1 shows that the time needed to perform a schedule through a Production/ Maintenance negotiation increases exponentially with the number of activities that can be performed on each machine of the workshop. Nevertheless, if the routings do not use the 'activity' concept and if no maintenance activity is considered, the time required to perform a schedule remains reasonable and it can be compared with the time taken by market schedulers. The reason is that in this example the MOs alone do not represent an important load for the workshop: when the best machine that offers to perform an activity is chosen according to the cost and delay objectives, this choice is seldom set into question since most of the orders can be delivered on time. The time needed to perform the production schedule alone increases in that case almost linearly when the number of activities that can be performed by a machine increases.

On the other hand, the manufacturing activities set new tight constraints. Each time a maintenance activity is in conflict with a manufacturing operation, the choice of the machine is set into question if the conflict solving does not allow total satisfaction of the two partners. This case occurs very often and, consequently, the management of the degrees of freedom created by the activities makes the processing time increase exponentially. The increase of the processing time can be limited if thresholds are set on the satisfaction degrees of the manufacturing and maintenance operations, the machine allocation only being set into question if the satisfaction decreases under these thresholds.

\subsection{Test conditions and results}

The interest of a negotiation between Production and Maintenance depends very much on the workshop and routings characteristics, e.g. if the machines do not have 
an important load, planning the maintenance operation first at their optimal location, then planning the manufacturing operations around these maintenance activities should not make an important difference with the negotiated schedule. On the other hand, the global satisfaction provided by negotiation increases when real conflicts occur, which requires that the workshop has an important load and that degrees of freedom do exist.

These are the main characteristics having an influence on the interest of negotiation.

- Interval of time between two preventive maintenance activities: a preventive maintenance operation performed once a month is clearly less disturbing than if it occurs twice a day.

- Compared duration of the maintenance and manufacturing activities.

- Degree of freedom existing in the positioning of a maintenance activity, modelled by the hull of the fuzzy sets described in figure 4.

Production/maintenance negotiation should be of main interest when maintenance activities are long compared with manufacturing activities and when they are frequently performed. Moreover, the negotiation requires degrees of freedom: as a consequence, the result should be better if sufficient degrees of freedom exist on the positioning of the maintenance activities, i.e. if the hull of the fuzzy temporal windows is not too narrow.

To check these assumptions, we have defined a set of tests combining different categories of the previous characteristics. Three categories have been defined for each characteristic, standing for 'low', 'average' and 'important' and they are defined as follows.

- Frequency of Maintenance (FM) activities:

- Low: maintenance activity every 4 days.

- Medium: maintenance activity every 2 days.

- High: one maintenance activity per day.

- Maintenance Duration (MD): each duration is drawn according to a uniform law in the range:

- Low: average duration: $\bar{d}=60 \mathrm{mn}, d_{\min }=30 \mathrm{~min}, d_{\max }=90 \mathrm{mn}$.

- Medium: $\bar{d}=180 \mathrm{mn}, d_{\min }=90 \mathrm{~min}, d_{\max }=270 \mathrm{mn}$.

- High: $\bar{d}=360 \mathrm{mn}, d_{\min }=180 \mathrm{~min}, d_{\max }=540 \mathrm{mn}$.

- Constraint on Maintenance Operation Positioning (MP): this constraint is low when the size of the maintenance fuzzy window is large, and conversely:

- Low: kernel $=120 \mathrm{mn}$, hull $=600 \mathrm{mn}$.

- Medium: kernel $=60 \mathrm{mn}$, hull $=240 \mathrm{mn}$.

- High: kernel $=30 \mathrm{mn}$, hull $=120 \mathrm{mn}$.

A test denoted 'HHM' means for instance that the frequency of maintenance duration is high, the maintenance duration is high and the constraint on maintenance positioning is medium.

When combining these values, some test benches are clearly not relevant, e.g. performing twice-a-day maintenance operations with an average duration of 3 hours sets constraints that are not possible to satisfy and, moreover, which are irrelevant. 
Consequently, we have selected the 20 sets of hypothesis described in figure 9 among the 27 possible ones.

Finally, for each set of hypothesis, 95 schedules have been performed on different workshops, generated as follows.

- Workshop composed of five machines is considered.

- Ten routings composed of five operations each have been generated with predefined processing times associated with each operation. Each processing time has a duration of between 30 and $135 \mathrm{mn}$.

- Forty MOs are generated with predefined due dates, each being associated with one of the routings, randomly drawn. To each operation is then associated a resource, randomly drawn among the machines of the workshop.

To compare the Production/Maintenance negotiation with a classical scheduling technique, we have also performed two other schedules for each test:

- one in which production activities have systematically priority on maintenance activities;

- the other in which maintenance activities are scheduled first.

We have mainly analysed in the following the lateness of the MOs and the degree of satisfaction provided by the location of maintenance activities.

Figure 9 shows the average lateness of the MOs in the 20 examples according to the three types of schedule performed (the percentage of late orders gives very similar graphs). The lateness of the MOs is of course minimum when production has priority on maintenance (dark front bars) and do not depend on the test conditions which only concern maintenance parameters. When maintenance has priority (white rear bars), it can be checked that the size of the fuzzy window has no influence (maintenance activities are positioned as early as possible): the results are always similar for three consecutive tests. One can also verify that the lateness increases with maintenance frequency and duration. We can also verify in figure 9 that negotiation provides intermediate results between priority to maintenance and priority to production. The benefit for the lateness is not very high in the very tight cases (tests 3-5), but is more important in the 'intermediate' tests (tests 6-14). Of course, we shall see

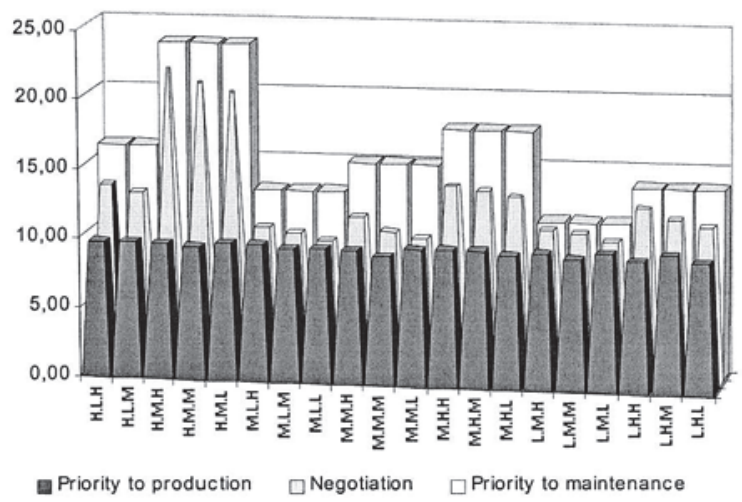

Figure 9. Average lateness of the manufacturing orders. 
below that this improvement is obtained at the price of a less good positioning of the maintenance activities.

Figure 10 shows the average degree of satisfaction provided by the positioning of the maintenance activities. As expected, this degree is equal to 1 when maintenance has priority (white rear bars). Figure 10 shows that the satisfaction of the positioning is drastically improved by negotiation: the average positioning satisfaction on all the tests jumps from 0.3 (priority to production) to 0.85 (compromise). The average

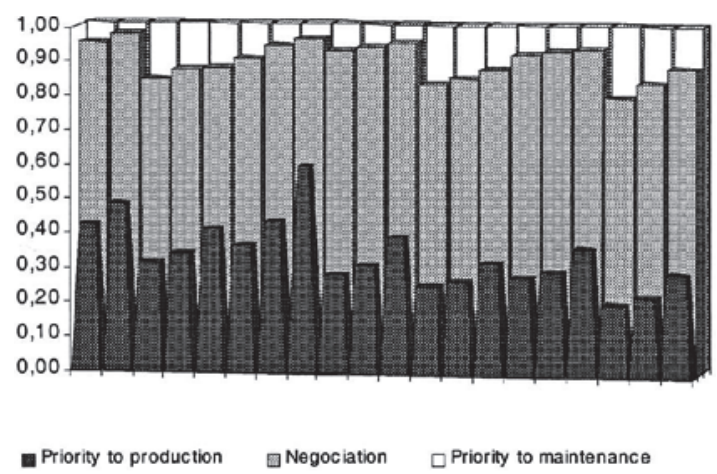

Figure 10. Satisfaction provided by the positioning of maintenance activities.

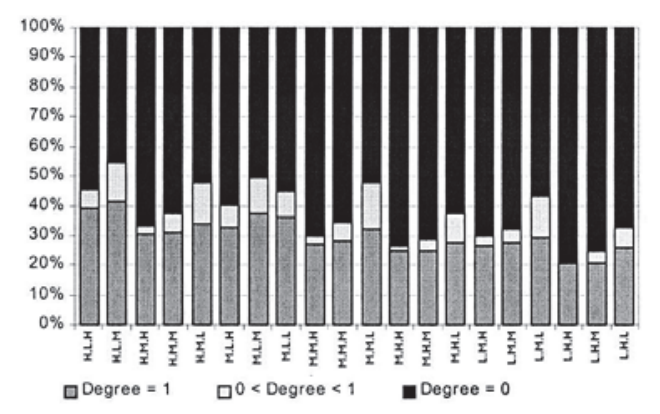

(a)

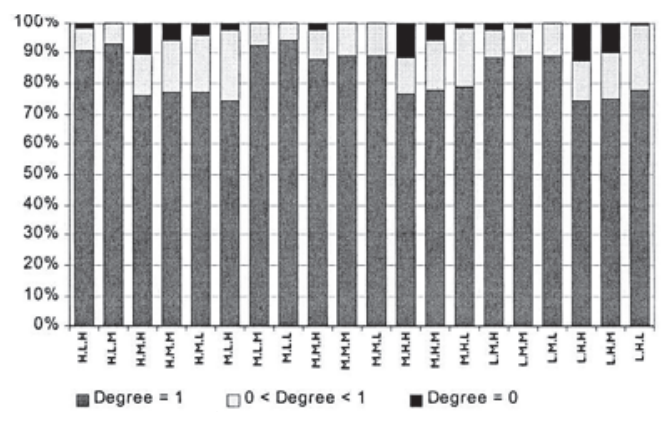

(b)

Figure 11. (a) Distribution of maintenance activities satisfaction: priority to production. (b) Distribution of maintenance activities satisfaction: negotiation. 
satisfaction increases when the constraint on the maintenance activities position decreases (size of the fuzzy window increasing), which is denoted by increasing steps in three consecutive examples. The domain of interest of compromises can be verified by correlating figures 9 and 10: on tests $3-5$, which denote the most tighten cases, the increase in the maintenance activity positioning requires one to accept an almost maximum lateness of the MOs. On tests that present higher degrees of freedom (e.g. tests 6-14), the increase of MOs lateness remains limited while the satisfaction of the maintenance activities positioning is drastically improved. As suggested by the good sense, negotiation is particularly interesting when each partner has sufficient degrees of freedom for negotiation

Figures $11 \mathrm{a}$ and $11 \mathrm{~b}$ show the distribution of the satisfaction degrees provided by the maintenance activities position in case of priority to production (figure 11a) and negotiation (figure 11b). In the first case, the majority of the maintenance activities have a positioning providing a null satisfaction, while almost $85 \%$ of the maintenance activities have fully satisfactory positioning in case of negotiation.

\section{Conclusion}

The planning of preventive maintenance activities in given time intervals may disturb the schedule of the MOs, but is mandatory to insure the long-term availability of the production system, especially when maintenance activities are often required. These maintenance activities may be performed on the machine itself, but they may also concern tools, changes of baths in thermal treatments or cleaning activities in dirty processes, like painting or offset printing. In that context, the multiagent paradigm may provide an implementation framework allowing one to model the negotiation process between the maintenance and production functions. We have shown here that fuzzy logic provides interesting facilities for modelling the degrees of freedom of the negotiation in a quite natural way. New developments are now in progress in order to define negotiation frameworks for other functions. Two types of cooperation seem promising at this moment: workforce management/production, especially in the context of annualized hours, which is now increasingly more common in France, and inventory control/production. Since the RAMSES II environment can coordinate the schedules of several workshops, the latter gives one the opportunity to address Supply Chain problems with a negotiation point of view, which opens new areas of investigation.

\section{References}

Archimède, B. and Coudert, T., 2001, Reactive scheduling using a multi-agent model: the SCEP framework. Engineering Applications of Artificial Intelligence, 14, 667-683.

Ashayeri, J, Teelen, A. and Selen, W., 1996, A production and maintenance planning model for the process industry. International Journal of Production Research, 34, $3311-3326$

Ayel, J., 1994, Concurrent decisions in production management. Journal of Integrated Computer-Aided Engineering (Special Issue on 'AI in Manufacturing and Robotics').

BarnetT, K. W. and BLUNDELL, J. K., 1981, Trade demarcations in maintenance: determination of optimum crew sizes by the Monte-Carlo simulation technique. Terotechnica, 2 , $147-155$.

Basker, B. A. and Husband, T. M., 1982, Simulating multi-skill maintenances: a case study. Maintenance Management International, 3, 173-182.

Brandolese, M., Franci, M. and Pozzetti, A., 1996, Production and maintenance integrated planning. International Journal of Production Research, 34, 2059-2075. 
Coudert, T., Archimède, B. and Grabot, B., 1999, Un système multi-agents pour la coopération Production/maintenance. In G. Habchi and A. Haurat (eds), Actes de la deuxième conférence francophone de Modélisation et SIMulation (MOSIM'99), 6-8 October (Annecy: SCS International).

De Carvalho, S. V. and Noyes, D., 1996, A Markov model for the optimization of a maintenance system with a performance constraint. In Annals of the Brazilian Symposium on Operations Research, Rio de Janeiro, Brazil, 26-28 July, pp. 1355-1360.

Deniaud, S., Zerhouni, N., El Moudni, A. and Morel, F., 1999, Planification de la maintenance préventive d'une ligne de fabrication d'Alstom. APII-JESA, 33, 227-250.

Erschler, J., Roubellat, F. and Vernhes, J. P., 1976, Finding some essential characteristics of the feasible solutions for a scheduling problem. Operations Research, 24, 774-783.

Fox, M. S., 1987, Constraint-directed Search: A Case Study of Job-shop Scheduling (London: Pitman).

Gits, C. W., 1994, Structuring maintenance control systems. International Journal of Operation and Production Management, 14, 5-17.

Graвот, B., 1998, Objective satisfaction assessment using neural nets for balancing multiple objectives. International Journal of Production Research, 36, 2377-2395.

Hammer, M. and Champy, J., 1994, Reengineering the Corporation: A Manifesto for Business Revolution (New York: HarperBusiness).

IsHII, H., 2000, Scheduling problems with fuzzy constraints. In R. Slowinski and M. Hapke (eds), Scheduling Under Fuzziness (Heidelberg: Physica).

Kelly, C. M., Mosier, C. T. and Mahmoodi, F., 1997, Impact of maintenance policies on the performance of manufacturing cells. International Journal of Production Research, 35, 767-787.

Kerr, R. M. and Walker, R. N., 1989, A job shop scheduling system based on fuzzy arithmetic. In Proceedings of the 3rd International Conference on Expert Systems and the Leading Edge in Production and Operation Management, Hilton Head Island, SC, USA.

LiU, J. and SyCARA, K. P., 1994, Distributed problem solving through coordination in a society of agents. Proceedings of the 13th International Workshop on DAI, Seattle, WA, USA, July.

Maturana, F., Shen, W. and Norrie, D. H., 1999, Metamorph: an adaptive agent-based architecture for intelligent manufacturing. International Journal of Production Research, 37, 2159-2173.

Miyashita, K., 1998, CAMPS: a constraint-based architecture for multiagent planning and scheduling. Journal of Intelligent Manufacturing, 9, 145-154.

Parunak, H. V. D., 1987, Manufacturing experience with the contract net. In M. N. Hunds (ed.), Distributed Artificial Intelligence (London: Pitman), pp. 285-310.

Paté-Cornell, M. E., Lee, H. L. and Tagaras, G., 1987, Warning of malfunction: the decision to inspect and maintain production process on schedule on or demand. Management Science, 3, 1277-1290.

Paz, N. M. and LeIGH, W., 1994, Maintenance scheduling: issues, results and research needs. International Journal of Operations and Production Management, 14, 47-69.

Rishel, T. D. and CHR ISTY, D. P., 1996, Incorporating maintenance activities into production planning; integration at the master schedule versus material requirement level. International Journal of Production Research, 34, 421-446.

SaAd, A., Kawamura, K. and Biswas, G., 1996, Performance evaluation of contract netbased heterarchical scheduling for flexible manufacturing systems. International Journal of Automation and Soft Computing [Special Issue on 'Intelligent Manufacturing Planning and Shopfloor Control'].

Sanmarti, E., Espuna, A. and Puigjaner, L., 1997, Batch production and preventive maintenance scheduling under equipment uncertainty. Computer Chemical Engineering, 21, $1157-1168$.

Shen, W. and Norrie, D. H., 1999, Agent-based systems for intelligent manufacturing: a state of the art survey. Knowledge and Information Systems, 1, 129-156.

Smith, R. G., 1980, The contract-net protocol: high level communication and control in a distributed problem solver. IEEE Transactions on Computers, 29, 1104-1113. 
Sмiтh, S. F., 1992, Knowledge-base production management: approaches, results and prospectives. Production Planning and Control, 4, 350-380.

Sohier, C., Denis, B. and Lesa Ge, J. J., 1998, Applying eco problem solving to the control of an adaptive manufacturing cell. Proceedings of CESA'96 Multiconference on Computational Engineering in systems applications, Lille, France, 9-12 July.

Swanson, L., 1999, The impact of new production technologies on the maintenance function: an empirical study. International Journal of Production Research, 37, 849-869.

Tranvouez, E., Espinasse, B. and Chirac, J. P., 1998, A multi-agent based scheduling system: a cooperative and reactive approach. Proceedings of the 9th Symposium on Information Control in Manufacturing, Nancy-Metz, France, 22-24 June, Advances in Industrial Engineering, 3, IFAC.

Weinstein, L. and CHUnG, C. H., 1999, Integrating maintenance and production decisions in a hierarchical production planning environment. Computers and Operations Research, 10-11(26), 1059-1074.

Zülch, G., GROBEL, T. and Jonsson, U., 1994, Indicators for the evaluation of organizational performance. IFIP WG 5.7 workshop on Benchmarking-Theory and Practice, Trondheim, Norway, 16-18 June. 